\title{
EDITORIAL
}

\section{When antibiotic treatment fails}

\author{
Matteo Bassetti ${ }^{1,5^{*}}$ (D), Josè Garnacho Montero ${ }^{2}$ and José Artur Paiva $a^{3,4}$
}

(c) 2017 Springer-Verlag GmbH Germany and ESICM

What to do when antimicrobial treatment fails and when and how to define it? These are three very common questions arising when clinicians use antibiotics. Categorization of responders and non-responders early in the course of antibiotic treatment has a meaningful relationship to outcomes that is important to patients, hospitals and payers [1].

No consensus statements on the definition of treatment failure have been published [2]. The detection of treatment failure is mostly based on objective clinical criteria (Fig. 1), but also on the less well-defined subjective decisions of the treating physicians.

Assessment of response should be performed early enough to allow successful rescue therapy, but not too early, to allow evidence of clinical response. Although time to response depends on the site of infection, on the pathogen and on the immunological status of the patient, and although different time-points for assessment may be necessary for different symptoms and signs [3], 48-72 h of adequate therapy are usually needed.

Response to treatment of an infection can be assessed using both clinical, biochemical and microbiological parameters. Clinical parameters include symptoms and signs (e.g., a decrease in fever, tachycardia or confusion), laboratory values (e.g., decreasing leukocyte count, PCT (procalcitonin) or CRP (C-reactive protein)), organ function markers (reduction or cessation of vasopressors, improvement of hypoxemia among others) and radiologic findings (e.g., size reduction of an abscess, improvement in lung opacities). Although radiologic criteria are commonly used in assessing response to infectious disease therapy, radiologic improvement can frequently lag behind clinical improvement, and routine radiographic

\footnotetext{
${ }^{*}$ Correspondence: matteo.bassetti@asuiud.sanita.fvg.it;

matteo.bassetti@uniud.it

${ }^{5}$ Clinica Malattie Infettive, Azienda Sanitaria Universitaria Integrata,

Presidio Ospedaliero Universitario Santa Maria della Misericordia, Piazzale

Santa Maria della, Misericordia 15, 33100 Udine, Italy

Full author information is available at the end of the article
}

follow-up of all infections is not always necessary. Clinical improvement is good indicator of therapy response; however, as Luna et al. suggested, most of the traditional measures of infection such as radiographic infiltrate, amount and quality of secretions, fever and high white cell count (WCC) are poor predictors of clinical response to therapy, whereas a more specific physiologic variable, such as the $\mathrm{PaO}_{2}$-to- $\mathrm{FiO}_{2}$ ratio, can much more accurately differentiate between responders and nonresponders [4]. Several weeks or even months may be required before chest radiography or computed tomography shows complete resolution of an infiltrate.

Bacteremia is the most common scenario in which microbiological response is closely assessed because clearance of the bloodstream is as important as clinical improvement. Persistent bacteremia can often be the only clue to the presence of an inadequately treated source or to the existence or development of complicated endovascular infection (such as endocarditis or an intravascular device infection). Persistent bacteremia can also be associated with the emergence of antimicrobial resistance and should always be investigated. The combined use of MALDI-TOF and antimicrobial stewardship and the judicious use, in selected populations, of molecular techniques allow earlier optimization of antimicrobial therapy [5].

Sustained defervescence, clinical stability $[6,7]$ and reduction of sequential organ failure assessment (SOFA) score comparing the day of re-assessment with the day antibiotic treatment is started [8], in a 48-72-h timeframe, are the variables that are considered in several studies and seem to better reflect clinical response.

Although CRP and PCT kinetics in the first 3-4 days of therapy may help to identify patients with a complicated course [9], a biomarker-based strategy failed to improve outcome as compared to usual care. Therefore, a strategy of antibiotic escalation based exclusively on PCT or CRP results should be discouraged [10]. 


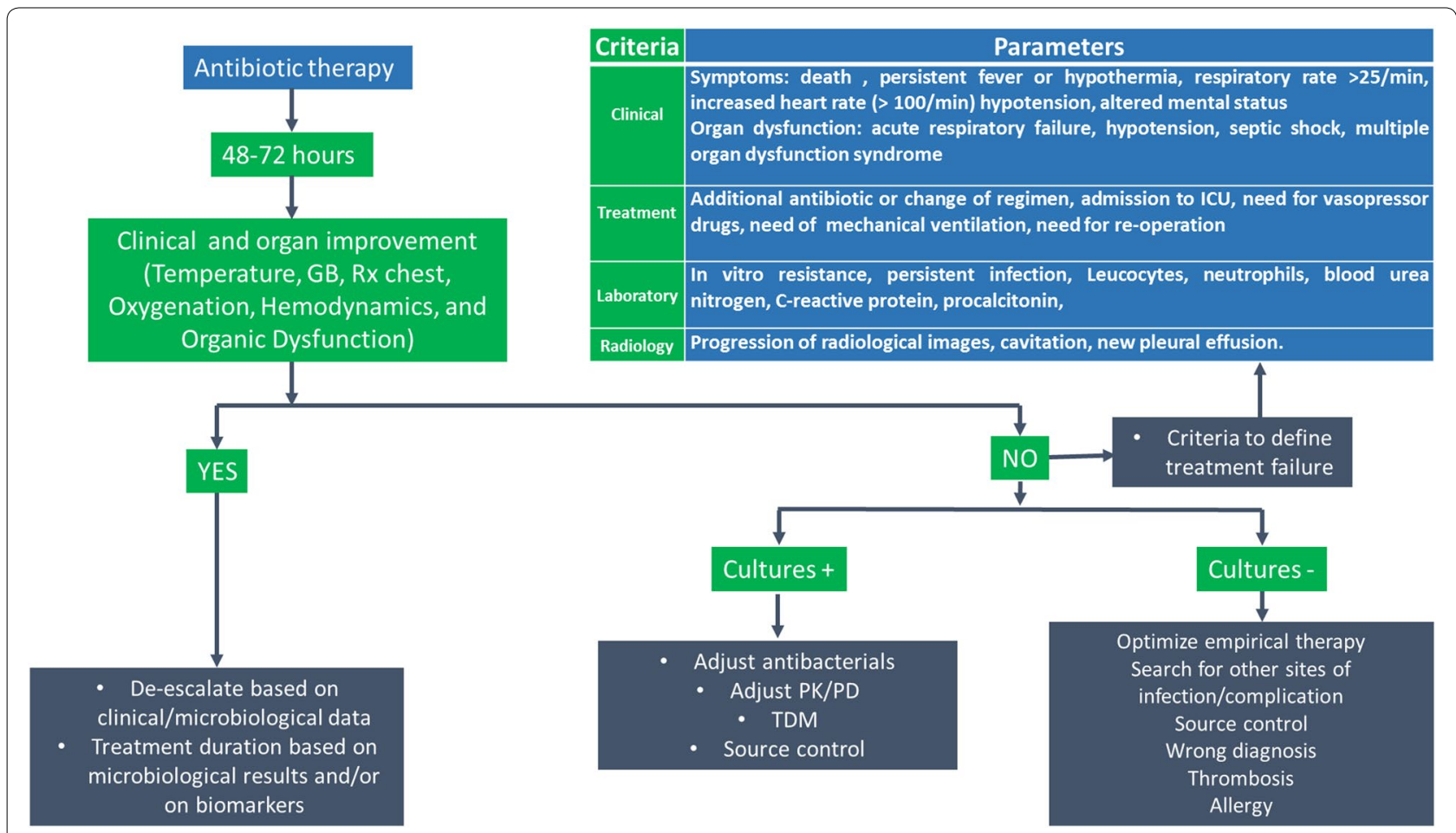

Fig. 1 Algorithm to define antibiotic failure and what to do in case of failure

In the face of absence of response to empirical antibiotic therapy, microbiological results must be considered and if first-line therapy was inappropriate, the antibiotic should be changed swiftly. In fact, the spread of multidrug-resistant infections is leading to an increase in empirical antibiotic treatment failure.

However, antibiotic inappropriateness is neither the only nor the more common cause of therapy failure [11]. If microbiological results show that an antibiotic regimen (that is now defined "targeted") is appropriate or if they are inconclusive, several other causes of therapy failure must be considered, namely: wrong diagnosis (non-infectious disease), development of infectious complication, persistence of inflammation, inadequate source control, antibiotic pharmacokinetic and pharmacodynamic (PK/ $\mathrm{PD})$ issues and host immunosuppression [12]. Failure to respond to antibiotics includes also the emergence of resistant organisms, superinfections and drug interactions. The most common mistake made with apparent antibiotic failure is to change or add antibiotics. The most important strategy is to analyze the cause of the antibiotic failure by careful evaluation and use of appropriate diagnostic tests to avoid needless, and expensive and potentially dangerous antimicrobial therapy (see Fig. 1).

Apart from in vitro antimicrobial activity and achievement of adequate serum levels, the efficacy of antimicrobial agents depends on their capacity to achieve a concentration equal to or greater than the minimum inhibitory concentration (MIC) at the site of infection. Antimicrobial concentrations attained at some sites [e.g., ocular fluid, cerebral spine fluid (CSF), abscess cavity, prostate and bone] are often much lower than serum levels. For example, first- and second-generation cephalosporins and macrolides do not cross the blood-brain barrier and are not recommended for CNS infections. Daptomycin, an excellent bactericidal agent against gram-positive bacteria, is not useful for treatment of pneumonia (e.g., pneumococcal pneumonia) because it is inactivated by a lung surfactant The presence of foreign bodies at the site of infection also affects antimicrobial activity. Other potential causes of treatment failure include the wrong dosaging of antimicrobials, particularly important in certain patient populations such as the critically ill. Unfortunately, monitoring of antimicrobial drug concentrations is not available routinely for most compounds in most of the hospitals, and remains a sort of a "dream" for many physicians. These tools would enable intensive care specialists to adjust for the continuous changes in volume of distribution and drug clearance that occur in the critically ill. The increased volume of distribution that follows and glomeral hyperfiltration associated with severe sepsis and septic shock lead 
to low plasma concentrations of antimicrobials. In turn, attempts to correct for underdosing may be offset by the development of renal or hepatic dysfunction.

Antimicrobial therapy is usually, but not always, the most important therapy for infectious diseases; however, there are many "non-antimicrobial therapies" that may avoid treatment failure. The best-recognized example of non-antimicrobial therapy in the treatment of infections is "source control", namely the use of operative drainage or debridement. This procedure is useful when the organism burden is very high or in the management of abscesses, for which the penetration and activity of antimicrobial agents are often inadequate. Other therapies used in the treatment of infectious diseases involve modulating the host inflammatory response to infection. Systemic corticosteroids, thought to act by decreasing the deleterious effects of the host inflammatory response, have been found beneficial when used in conjunction with antimicrobial therapy for the treatment of bacterial meningitis, tuberculous meningitis and pneumocystis pneumonia in patients with AIDS. Temporary discontinuation or dose reduction of immunosuppressive agents is often required for successful treatment of infections in organ transplant recipients or patients with rheumatologic disorders. Intravenous immunoglobulin therapy, which acts to neutralize toxin produced by the bacteria, can be used in addition to surgical debridement and antimicrobial therapy in the treatment of necrotizing fasciitis caused by group A streptococci and severe toxic shock syndrome. Some of these interventions lack a strong evidence base but are often recommended by experts on the basis of clinical experience.

In conclusion, we believe that adequate and timely assessment of response to treatment and awareness of antibiotic failure may potentially increase the quality of treatment and improve outcomes. New molecular and rapid diagnostic tools may play a significant role in reducing the time for defining failure, for adjusting treatment and, consequently, for improving prognosis of serious infections.

\section{Author details}

${ }^{1}$ Infectious Diseases Clinic, Department of Medicine, University of Udine and Azienda Sanitaria Universitaria Integrata, Udine, Italy. ${ }^{2}$ Intensive Care Clinical Unit, Virgen Macarena University Hospital, Seville, Spain. ${ }^{3}$ Department of Emergency and Intensive Care, Centro Hospitalar São João, Faculty of Medicine, University of Porto, Porto, Portugal. ${ }^{4}$ Grupo de Infecçao e Sépsis, Porto,
Portugal. ${ }^{5}$ Clinica Malattie Infettive, Azienda Sanitaria Universitaria Integrata, Presidio Ospedaliero Universitario Santa Maria della Misericordia, Piazzale Santa Maria della, Misericordia 15, 33100 Udine, Italy.

\section{Compliance with ethical standards}

\section{Conflicts of interest}

Dr. Bassetti has participated in advisory boards and/or received speaker honoraria from Achaogen, Angelini, Astellas, AstraZeneca, Bayer, Basilea, Gilead, Menarini, MSD, Pfizer, The Medicine Company, Tetraphase and Vifor. Dr Garnacho-Montero has received speaker honoraria and a research grant from Astellas and MSD.

Received: 22 September 2017 Accepted: 9 October 2017

Published online: 14 October 2017

\section{References}

1. Talbot GH (2014) The early response end point in clinical trials not just for FDA anymore? Infect Dis Clin Pract 22(6):307-308

2. Sanchez Garcia M (2009) Early antibiotic failure. Int J Antimicrob Agents 34(S14):S19

3. Timsit JF, de Kraker MEA, Sommer H, Weiss E, Bettiol E et al (2017) Appropriate endpoints for evaluation of new antibiotic therapies for severe infections: a perspective from COMBACTE's STAT-Net. Intensive Care Med 43:1002-1012

4. Luna CM, Blanzaco D, Niederman MS et al (2003) Resolution of ventilatorassociated pneumonia: prospective evaluation of the clinical pulmonary infection score as an early clinical predictor of outcome. Crit Care Med 31:676-682

5. Bassetti M, Poulakou G, Ruppe E, Bouza E, Van Hal SJ, Brink A (2017) Antimicrobial resistance in the next 30 years, humankind, bugs and drugs: a visionary approach. Intensive Care Med. doi:10.1007/s00134-017-4878-x

6. Talbot GH, Powers JH, Fleming TR, Siuciak JA, Bradley J, Boucher H (2012) Progress on developing endpoints for registrational clinical trials of community-acquired bacterial pneumonia and acute bacterial skin and skin structure infections: update from the Biomarkers Consortium of the Foundation for the National Institutes of Health. Clin Infect Dis 55:1114-1121

7. Joung MK, Lee JA et al (2011) Impact of de-escalation therapy on clinical outcomes for intensive care unit-acquired pneumonia. Crit Care 15(2):R79

8. Garnacho-Montero J, Gutierrez-Pizarraya A, Escoresca-Ortega A et al (2014) De-escalation of empirical therapy is associated with lower mortality in patients with severe sepsis and septic shock. Intensive Care Med 40:32-40

9. Seligman R, Meisner M, Lisboa TC et al (2006) Decreases in procalcitonin and $\mathrm{C}$-reactive protein are strong predictors of survival in ventilatorassociated pneumonia. Crit Care 10:R125

10. Jensen JU, Hein L, Lundgren B et al (2011) Procalcitonin-guided interventions against infections to increase early appropriate antibiotics and improve survival in the intensive care unit: a randomized trial. Crit Care Med 39:2048-2058. doi:10.1097/CCM.0b013e31821e8791

11. Menendez R, Cavalcanti M, Reyes S, Mensa J, Martinez R, Marcos M et al (2008) Markers of treatment failure in hospitalised community acquired pneumonia. Thorax 63:447-452

12. Gonçalves-Pereira J, Conceiçao C, Póvoa P (2013) Community-acquired pneumonia: identification and evaluation of nonresponders. Ther Adv Infect Dis 1(1):5-17 